\title{
Haptic discrimination of material properties by a robotic hand
}

\author{
Shinya Takamuku*, Gabriel Gómez**, Koh Hosoda*, and Rolf Pfeifer** \\ *Dept. of Adaptive Machine Systems, Graduate School of Engineering, Osaka University, Japan \\ \{shinya.takamuku, hosoda\}@ams.eng.osaka-u.ac.jp \\ **Artificial Intelligence Laboratory \\ Dept. of Information Technology, University of Zurich, Switzerland \\ Andreasstrasse 15, CH-8050 Zurich, Switzerland \\ \{gomez,pfeifer\}@ifi.unizh.ch
}

\begin{abstract}
One of the key aspects of understanding human intelligence is to investigate how humans interact with their environment. Performing articulated movement and manipulation tasks in a constantly changing environment, have proven more difficult than expected. The difficulties of robot manipulation are in part due to the unbalanced relation between vision and haptic sensing. Most robots are equipped with high resolution cameras, which images are processed by well established computer vision algorithms such as color segmentation, motion detection, edge detection, etc. However, the majority of robots have very limited haptic capabilities.

This paper presents our attempt to overcome this difficulties by: (a) using a tendon driven robotic hand with rich dynamical movements and (b) covering the hand with a set of haptic sensors on the palm and the fingertips, the sensors are based on a simplified version of an artificial skin with strain gauges and PVDF (polyvinylidene fluoride) films. The results show that if the robotic hand actively explores different objects using the exploratory procedures: tapping and squeezing, material properties such as hardness and texture can be used to discriminate haptically between different objects.
\end{abstract}

Index Terms - Haptic perception, tapping, squeezing, artificial skin, tendon driven robotic hand.

\section{INTRODUCTION}

Humans rely on multiple sensory modalities to estimate environmental properties. Both, the eyes and the hands can provide information about an object's shape, but in contrast to vision, the hands are especially adapted to perceive material properties such as texture, temperature, and weight. Manual haptic perception is the ability to gather information about objects by using the hands. The tactile properties of objects are processed by the somatosensory system, which uses information from receptors that respond to touch and vibration, body movement, temperature, and pain [1]. A baby's earliest explorations of himself and his environment are made using his sense of touch ( [2], [3]), his hands and mouth being the principal exploratory tools. [4] presented evidence for genuine haptic perception of material properties (including weight differences) by infants as young as three months old, although they did not explore the objects with hand movements specific to the properties in question (which is due to their inability to move the fingers independently). The stimuli consisted of objects which were all held with the same kind of grip, in order that they could not be discriminated on the basis of hand posture and in total darkness to avoid visual perception or cross modal associations, in other words to make the exploration purely haptic.

Infants acquire ability to haptically detect various object properties asynchronously, first to size or volume (3 months), followed by texture, temperature, and hardness (6 months), weight (6-9 months) [5].

Haptic exploration is a task-dependent activity, and when people seek information about a particular object property, such as size, temperature, hardness, or texture, they perform stereotyped exploratory hand movements or "exploratory procedures" [6]. The exploratory procedures used by adults to explore haptic properties are lateral motion (a rubbing action) for detecting texture; pressure (squeezing or poking) for encoding hardness; static contact for temperature; lifting to perceive the weight; enclosure for volume and gross contour information; and contour following for precise contour information as well as global shape [7].

In terms of robotic manipulation, a fully integrated force/tactile sensor has been developed by [8] for the "machand", as well as a technique to compute the pressure centroid and the associated ellipsoid during contact (see [9]). A dynamical model for viscoelastic pads useful to quantitatively characterize the behavior of materials used to cover robotic hands was presented by [10], a control approach, exploiting the relation between the stiffness and the applied load, was proposed by [11] in order to arbitrarily change the overall stiffness of a robot hand. Using the robot "Obrero", [12] has demonstrated the feasibility of the "tapping" exploration procedure by using a finger to tap objects and use the produced sound to recognize them. A self organized map was 


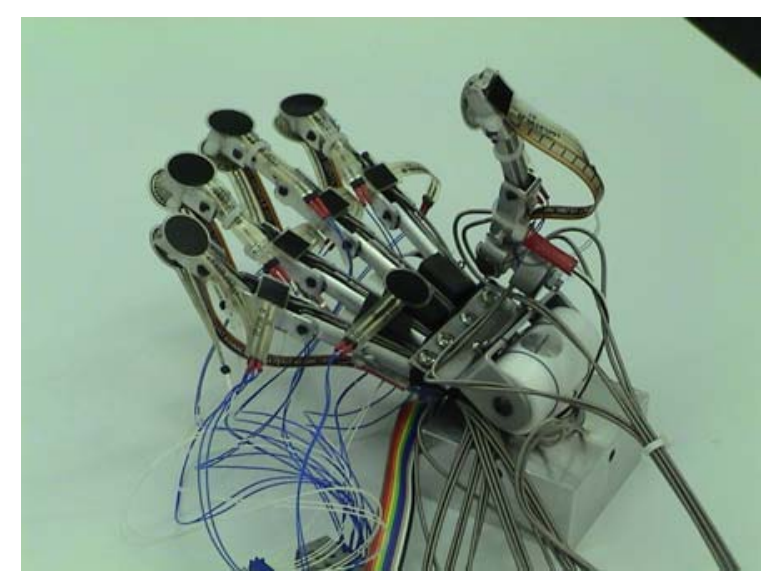

(a)

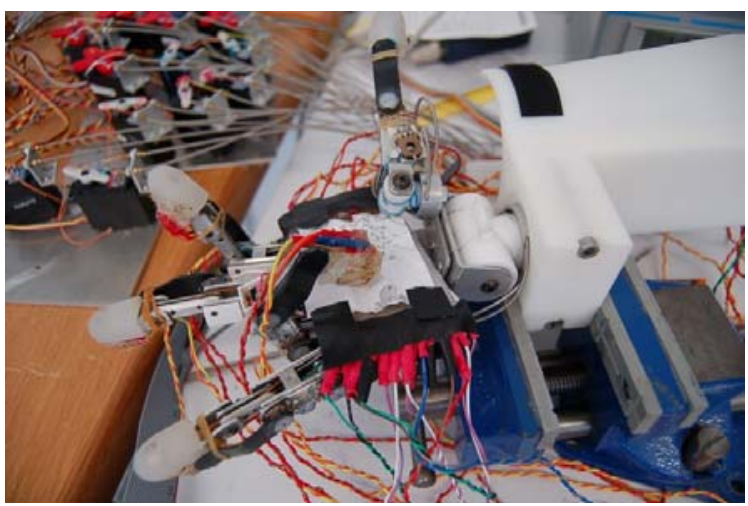

(b)

Fig. 1. Experimental setup. (a) tendon driven robotic hand (b) artificial skin with strain gauges and PVDF (polyvinylidene fluoride) films sensors mounted on the fingertips and the palm. The hand is exploring a piece of paper.

used by [13] to enable the "Babybot" to categorize 6 different objects plus the no-object condition. The network encoded not only the shape but intrinsic properties like weight.

In this paper we present our work with a tendon driven robot hand, the "Yokoi hand", developed by [14] covered with a set of haptic sensors on the palm and the fingertips, the sensors are based on a simplified version of an artificial skin with strain gauges and PVDF (polyvinylidene fluoride) films sensors developed by [15], [16]. In the following section we describe the tendon driven mechanism of our robotic hand and the position, type, and number of sensors covering it. In section III we specify the robot's task. In section IV we explain the different exploratory procedures. Then we present some experimental results as well as a discussion and future work.

\section{RoBOtIC SETUP}

Our robotic platform can be seen in Fig. 1a. The tendon driven robot hand is partly built from elastic, flexible and deformable materials (see [14]). The hand applies an adjustable

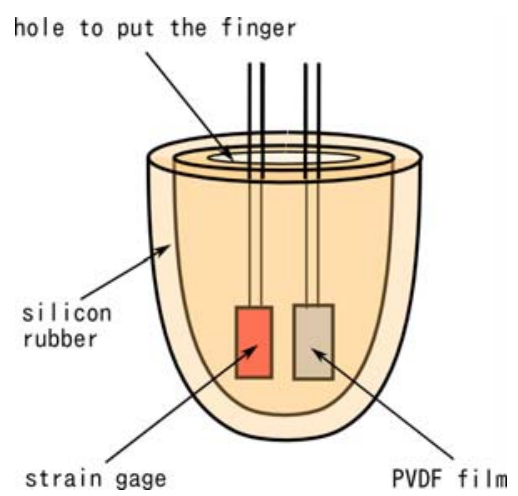

(a) Fingertip

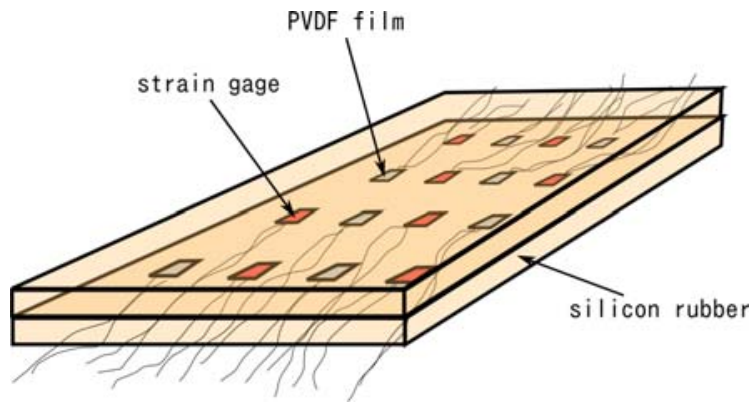

(b) Palm

Fig. 2. Sketches of the artificial skins

power mechanism develop by [17].

The robotic hand has 18 degrees of freedom (DOF) that are driven by 13 servomotors and has been equipped with three types of sensors: flex/bend, angle, and haptic.

\section{A. Bending and angle sensors}

For the flex/bend sensor, the bending angle is proportional to its resistance and responds to a physical range between straight and a 90 degree bend, they are placed on every finger as position sensors. Angle sensors in all the joints are provided by potentiometers.

\section{B. Haptic sensors}

Haptic sensors are based on strain gauges and PVDF (polyvinylidene fluoride) films sensors. The haptic sensors are located in the palm and in the fingertips as can be seen in Fig. 1. The artificial skin is made by putting strain gauges and PVDF (polyvinylidene fluoride) films between two layers of silicon rubber. The strain gauges detect the strain and work in a similar way as the Merkel cells in the human skin, whereas the PVDF films detect the velocity of the strain and corresponds to the Meissner corpuscles (MCs, see [18]) in the human skin. The PVDF films are expected to be more sensitive to the transient/small strain than the strain gauges. The shape of the artificial skin is modified to fit to the robotic hand. Sketches of the artificial skins for the fingers 


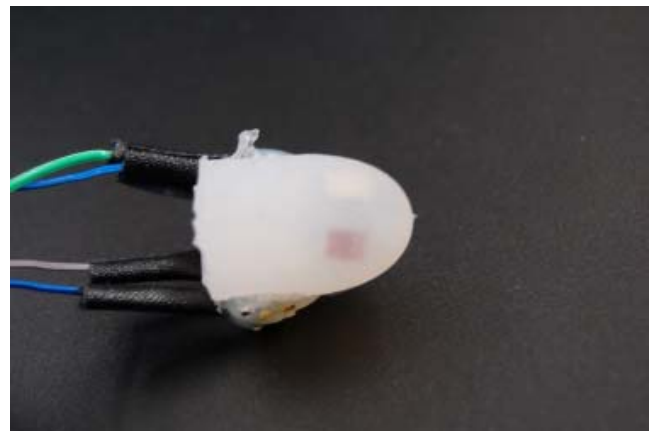

(a) Fingertip

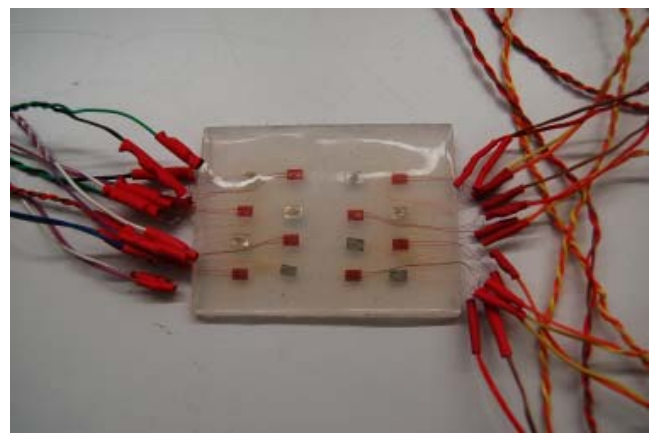

(b) Palm

Fig. 3. Pictures of the artificial skins

and the palm are shown in Fig. 2a and Fig. 2b respectively. Photographes for the corresponding skins are shown in Fig. 3a and Fig. 3b. In each fingertip there is one strain gauge and one PVDF film. In the palm there are four strain gauges and four PVDF films.

\section{Robot control}

We control the robot hand using a TITech ${ }^{\mathrm{TM}} \mathrm{SH} 2$ controller. The controller produces up to 16 PWM (pulse with modulation) signals for the servomotors and acquire the values from the bending and angle sensors. The motor controller receives the commands through an USB port.

Sensor signals from the strain gauges and the PVDF films are amplified and fed to a host computer via a CONTEC ${ }^{\mathrm{TM}}$ data acquisition card at a rate of $1.6 \mathrm{KHz}$.

\section{ROBOT TASK}

The robot performs two exploratory procedures with the ring finger, namely: squeezing, and tapping over seven different objects of different material properties as well as the no-object condition, each object was taped on the palm of the robot hand and explored during one minute. The objects can be seen in Fig. 4. The robotic hand performing a typical experiment can be seen in Fig. 1b.

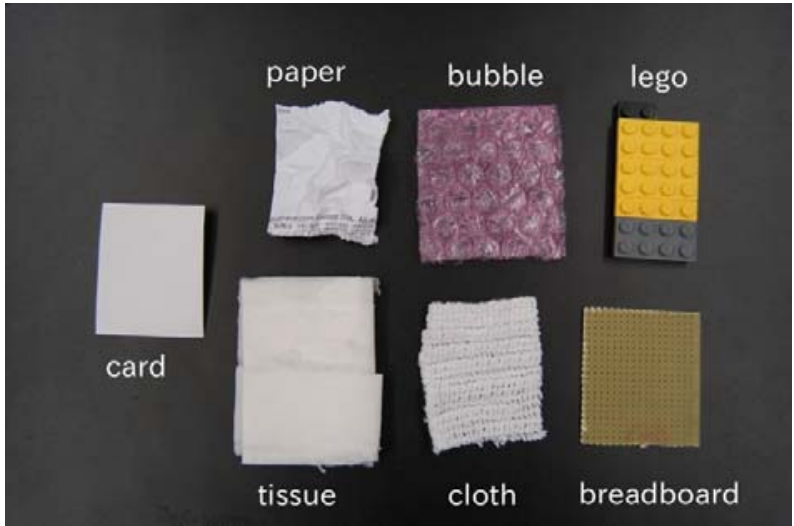

Fig. 4. Objects with different material properties.

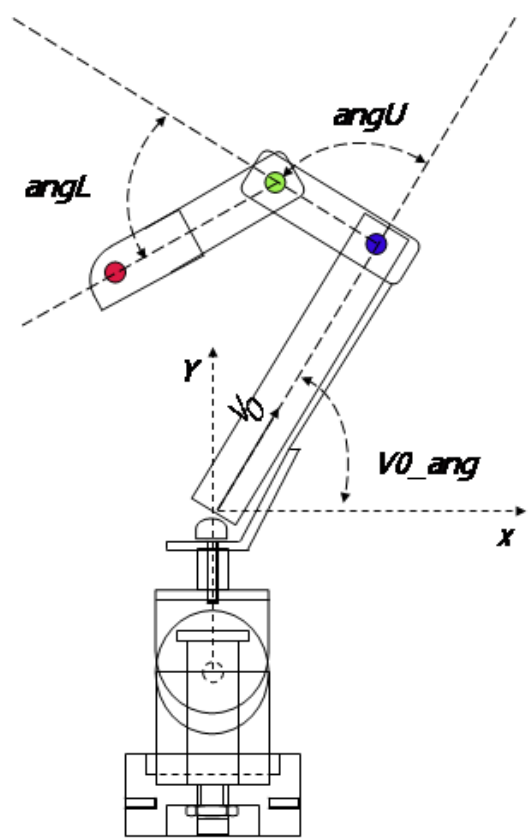

Fig. 5. Schematic of a finger. Positions of the fingertip (red marker), the middle hinge (green marker) and the base (blue marker)

\section{EXPLORATORY PROCEDURES}

The robotic hand actively explores different objects using the exploratory procedures: squeezing and tapping.

\section{A. Squeezing}

For the squeezing exploratory procedure, we drove both motors controlling the ring finger to the maximum angular position, thus making the finger to close over the palm as much as possible and squeezing the object, as described in 1.

$$
\operatorname{ang}_{i}(t)=\max A n g_{i}
$$




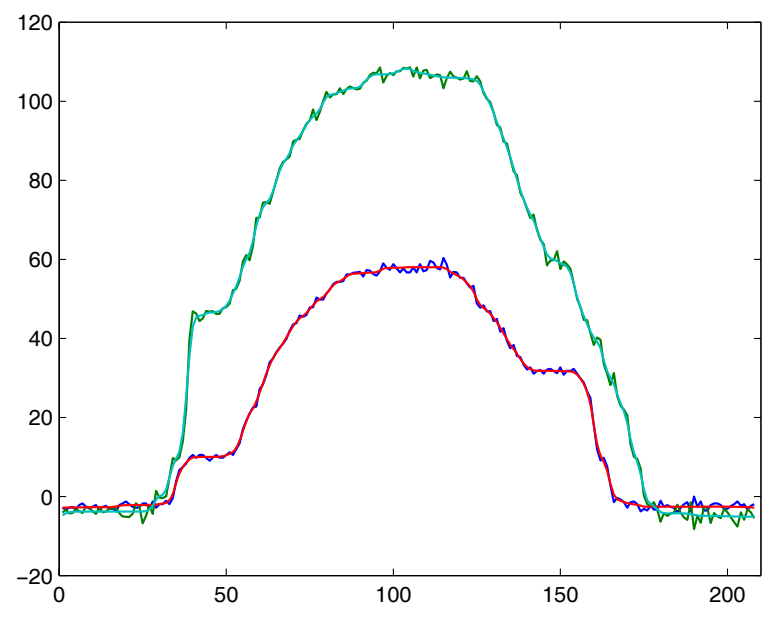

Fig. 6. Kinematics of the robotic hand.) sinusoidal position control. Upper plot is the angle between the middle hinge and the finger tip (angL), whereas the lower plot corresponds to the angle between the base of the finger and the middle hinge (angU).

Where:

- $a n g_{i}$ is the target angular positions of the i-th finger joint $\left(a n g_{L}\right.$ and $\left.a n g_{U}\right)$

- $\max A n g_{i}$ is the maximum angular position of the $\mathrm{i}$-th finger joint

\section{B. Tapping}

The tapping exploratory procedure was achieved by a sinusoidal position control of the ring finger that can be described as follows:

$$
\operatorname{ang}_{i}(t)=A_{i} \sin (\omega t+\phi)+B_{i}
$$

Where:

- $a n g_{i}$ is the target angular positions of the i-th finger joint $\left(a n g_{L}\right.$ and $\left.a n g_{U}\right)$.

- $A_{i}$ is the amplitude of the oscillation for the i-th finger joint

- $B_{i}$ is the set point of the oscillation (i.e., 60 degrees) for the i-th finger joint

- $\omega$ is the frequency of the oscillation.

- $\phi$ is the phase delay between the oscillation of the finger joints

Increasing and decreasing the position of the servo motors produced the pulling of the tendons, which made the fingers move back and forth, tapping the object over the palm. Fig. 6 shows the result of the motion of the finger during the no-object condition.

\section{RESUlTS}

Fig. 7 shows a time series of the results of typical squeezing experiments during $10 \mathrm{sec}$. There are 4 strain gauges

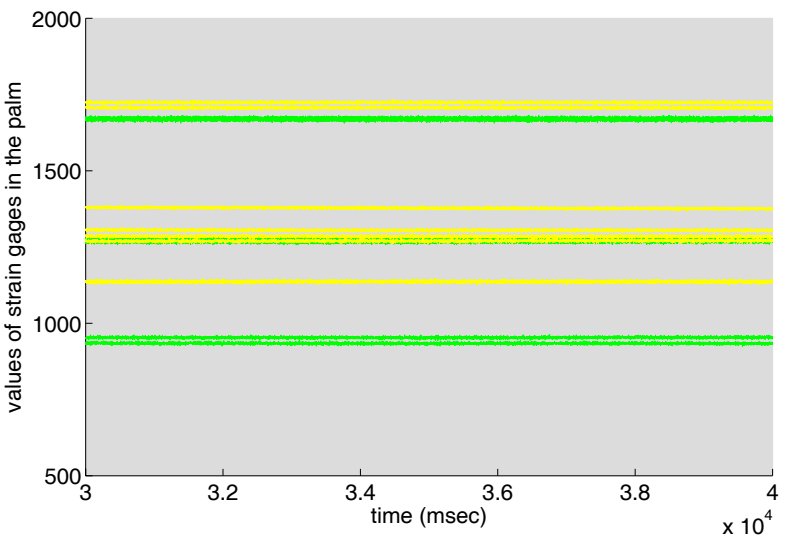

Fig. 7. Squeezing Exploratory procedure. Output of the four strain gauges located on the palm of the robotic hand while exploring a piece of tissue (yellow) and a circuit breadboard (light green) during $10 \mathrm{sec}$.

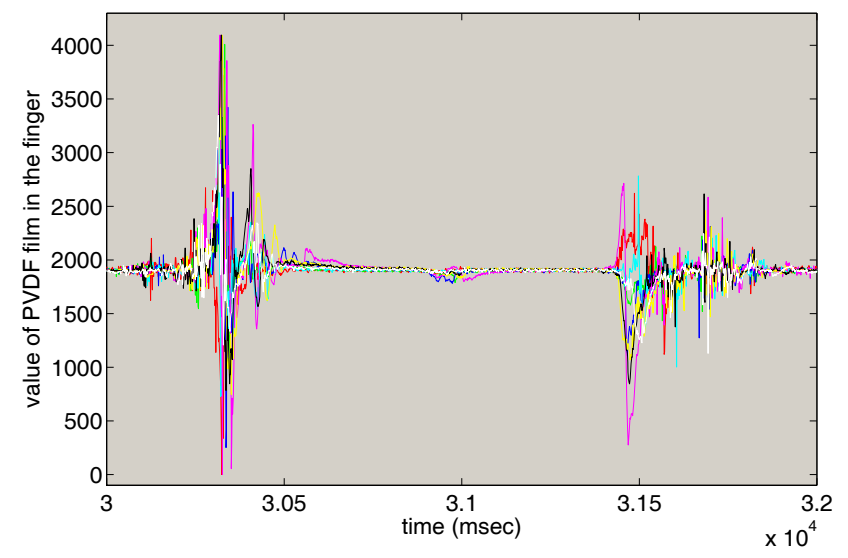

Fig. 8. Tapping exploratory procedure. Output of the PVDF film located on the fingertip of the ring finger while exploring the objects in Fig. 4 during 2 sec.

on the palm: the yellow lines represent the output of the strain gauges during the squeezing of a piece of tissue (soft material), whereas the light green lines represent the output of the strain gauges while squeezing a circuit breadboard (hard material). As can be seen the squeezing exploratory procedure can be used to distinguish the compliance (hardness of an object).

Fig. 8 shows a time series of a typical tapping experiment during $2 \mathrm{sec}$. There is one strain gauge and one PVDF film in the sensor located on the fingertip of the ring finger. The color correspondence is as follows: no-object condition(red), breadboard (light green), card (blue), lego (light blue), paper (pink), tissue (yellow), cloth (black), bubble (white). The larger output corresponds to the moment when the finger taps over the object and the smaller output corresponds to the moment when the finger is pull back and leaves the object. 


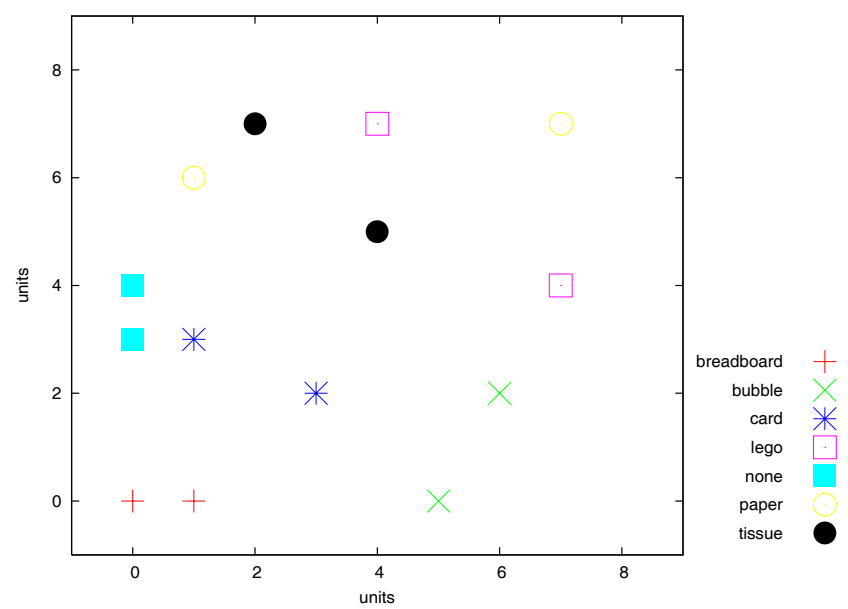

Fig. 9. Classification of seven objects plus the no-object condition by a SOM using the "Squeezing" exploratory procedure.

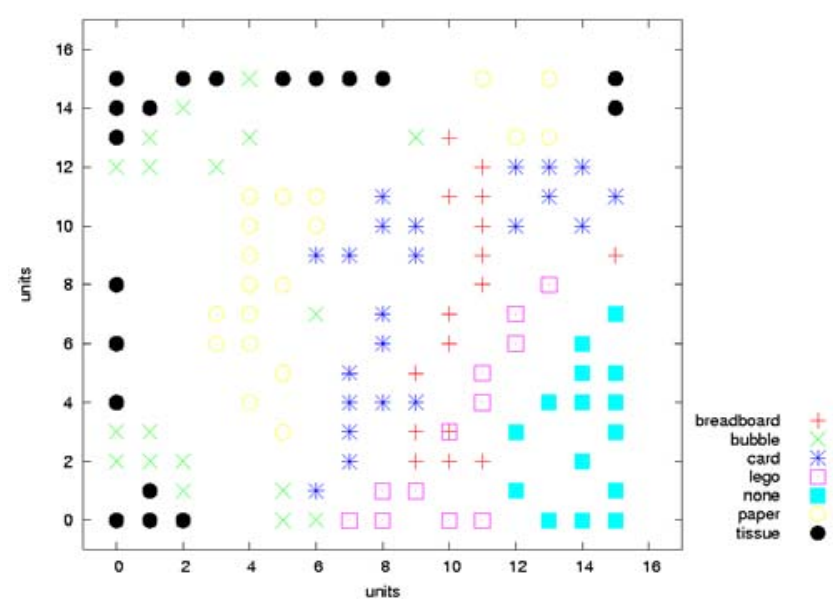

Fig. 10. Classification of seven objects plus the no-object condition by a SOM using the "Tapping" exploratory procedure.

A self organizing map was used to categorize the experimental data, we used the software package SOM_PAK version 3.1 [19], where the topology was a hexagonal lattice, the neighboring function type used was bubble.

Fig. 9 shows the result for the "squeezing" exploratory procedure, the input for the SOM was the average value of the four strain gauges in the palm and the size of the SOM was $8 \times 8$. As can be seen soft objects (e.g., tissue) are located in the middle, whereas hard objects (eg., circuit breadboard and lego) are distributed on the sides of the map.

For "tapping" the input was the sensory sequence of the PVDF film located in the finger and the results are shown in Fig. 10. The number of samples per object was 50 and the size of the SOM was increased to $16 \times 16$. As can be seen, the soft objects are located at the left and the upper part while the hard objects are distributed in the middle. The no-object condition is in the bottom right part. In other words, moving left and up in the map is being soft.

\section{DISCUSSION AND FUTURE WORK}

The research on haptic perception is certainly important to development and learning and our results show how the exploratory procedures: squeezing and tapping, can be used to recognize the hardness of an object as well as the no-object condition. Our robotic approach gives objective representations to the categories that we obtain from such exploratory behaviors. Such categories should be useful to discuss in more detail the haptic experience reported by infants or adults while exploring objects using the same type of exploratory procedures.

In the future we will introduce a "rubbing" exploratory procedure to encode texture and some friction features. Visual and proprioceptive information will be also included in order to make the categorization more robust. At the moment the artificial skin used in the palm is very flat and smooth, we would like to make it more closely to a human palm, with a concave shape and more rough in order to increase the contact surface with the objects and the possibility to stimulate the sensors easier.

\section{ACKNOWLEDGMENT}

This research was supported by the Swiss National Science Foundation project: "Embryogenic Evolution: From Simulation to Robotic Application," Nr.200021-109864/1 and the European Project: "ROBOTCUB: ROBotic Open Architecture Technology for Cognition Understanding and Behavior," Nr. IST-004370.

\section{REFERENCES}

[1] J. H. Kaas, "The functional organization of somatosensory cortex in primates," Ann Anat, vol. 175, p. 509518, 1993.

[2] A. Streri and Féron, "The development of haptic abilities in very young infants: From perception to cognition," Infant Behavior and Development, vol. 28, pp. 290-304, 2005.

[3] M. Molina and F. Jouen, "Manual cyclical activity as an exploratory tool in neonates," Infant Behavior and Development, vol. 27, pp. 4253, 2004.

[4] T. Striano and E. Bushnell, "Haptic perception of material properties by 3-month-old infants," Infant Behavior \& Development, vol. 28, p. 266289, 2005.

[5] E. W. Bushnell and J. P. Boudreau, "Motor development and the mind: The potential role of motor abilities as a determinant of aspects of perceptual development," Child Development, vol. 64, no. 4, pp. 10051021, August 1993.

[6] S. Lederman and R. Klatzky, "Hand movements: A window into haptic object recognition," Cognitive Psychology, vol. 19, p. 342368, 1987.

[7] S. J. Lederman and R. L. Klatzky, "Haptic exploration and object representation," in Vision and Action: The Control of Grasping, M. Goodale, Ed. New Jersey: Ablex, 1990, pp. 98-109.

[8] G. Cannata and M. Maggiali, "An embedded tactile and force sensor for robotic manipulation and grasping," in 5th IEEE-RAS International Conference on Humanoid Robots, 2005, pp. 80-85. 
[9] _ - "Processing of tactile/force measurements for a fully embedded sensor," in the IEEE International conference on multisensor fusion and integration for intelligent systems, Heidelberg, Germany, 2006, p. 160166

[10] L. Biagiotti, C. Melchiorri, P. Tiezzi, and G. Vassura, "Modelling and identification of soft pads for robotic hands," in IEEE/RSJ International Conference on Intelligent Robots and Systems (IROS 2005), 2005.

[11] L. Biagiotti, P. Tiezzi, G. Vassura, and C. Melchiorri, "Modelling and controlling the compliance of a robotic hand with soft finger-pads," Tracts in AdvancedRobotics, vol. 18, p. 5575, 2005.

[12] E. Torres-Jara, L. Natale, and P. Fitzpatrick, "Tapping into touch," in Fifth International Workshop on Epigenetic Robotics, Nara, Japan. July 22-24, 2005, 2005.

[13] L. Natale, G. Metta, and G. Sandini, "Learning haptic representation of objects." in In International Conference on Intelligent Manipulation and Grasping, Genoa - Italy, July 2004.

[14] H. Yokoi, A. Hernandez Arieta, R. Katoh, W. Yu, I. Watanabe, and M. Maruishi, Mutual Adaptation in a Prosthetics Application In Embodied artificial intelligence. Lecture Notes in Computer Science., F. Iida, R. Pfeifer, L. Steels, and Y. Kuniyoshi, Eds. Springer, ISBN: 3-540-22484-X, 2004, vol. 3139.

[15] Y. Tada, K. Hosoda, and M. Asada, "Sensing ability of anthropomorphic fingertip with multi-modal sensors," in 8th Conference on Intelligent Autonomous Systems (IAS8), March 2004., pp. 1005-1012.

[16] K. Hosoda, Y. Tada, and M. Asada, "Anthropomorphic robotic soft fingertip with randomly distributed receptors," Robotics and Autonomous Sys- tems, vol. 54, no. 2, pp. 104-109, 2006.

[17] Y. Ishikawa, W. Yu, H. Yokoi, and Y. Kakazu, "Research on the double power mechanism of the tendon driven robot hand," The Robotics Society of Japan, pp. 933-934, 1999.

[18] J. N. Hoffmann, A. G. Montag, and N. J. Dominy, "Meissner corpuscles and somatosensory acuity: The prehensile appendages of primates and elephants," THE ANATOMICAL RECORD PART A, vol. 281A, p. 11381147, 2004.

[19] T. Kohonen, J. Hynninen, J. Kangas, and J. Laaksonen, "Som_pak: The self-organizing map program package." Report A31. Helsinki University of Technology, Laboratory of Computer and Information Science, Espoo, Finland., Tech. Rep., 1996. 